\title{
SSI: The Stellar Seismic Indices Data Base ${ }^{\star}$
}

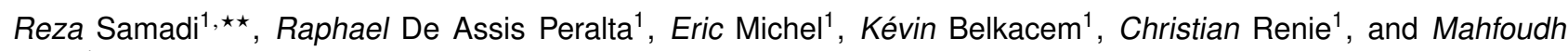 \\ Abed $^{1}$
}

${ }^{1}$ LESIA, Observatoire de Paris, CNRS UMR8109, Université Pierre et Marie Curie, Université Paris Diderot

\begin{abstract}
The Stellar Seismic Indices database (hereafter SSI) has been developed within the SPACEInn project funded under the UE Seventh Framework Programme (FP7). This data base aims at providing stellar seismic indices of solar-like oscillating stars as well as other stellar indices such as the main characteristics of stellar granulation. These indices are extracted using an automatic method, which is able to derive simultaneously the seismic and granulation parameters. This method was applied to almost all Kepler long-cadence light-curves and to almost all CoRoT targets observed in the faint fields. A total of about 320000 targets have been analyzed, among which seismic indices and granulation parameters have been extracted for about 18000 redgiants. In this article, we present the data base content, its interface, as well as some illustrative results.
\end{abstract}

\section{Stellar parameters extraction}

The seismic indices and stellar granulation parameters are extracted for red-giant stars by fitting their power density spectrum (PDS) using a semi-empirical model, which is illustrated in Fig. 1. The latter is composed of an activity component (green line), a granulation component (blue line), and an oscillation pattern (red line). More details are provided in de Assis Peralta et al [1]. Subsequently, three seismic indices are extracted from the fitted PDS:

- The peak frequency, $v_{\max }$ : it is defined as the frequency at which the oscillation spectrum maximum peaks in the PDS;

- The mean large separation, $\Delta v$ : this quantity corresponds to the mean frequency spacing between two consecutive p-modes (with same angular degree). It is derived using the Universal Pattern as proposed by [2];

- The height of the oscillation envelope, $H_{\mathrm{env}}$.

Together with the seismic indices, two parameters characterizing the stellar granulation background are also extracted from the PDS:

- The "e-folding time", $\tau_{\text {eff }}$, which corresponds to the time the granulation signal loose its coherence [e.g. 3, 4]. This time is also believed to represent the typical granule lifetime ;

- The variance of the integrated brightness fluctuations, $\sigma^{2}$, which corresponds to the total integrated energy within the granulation background.

\footnotetext{
${ }^{\star}$ http://ssi.lesia.obspm.fr

$\star \star$ e-mail: reza.samadi@obspm.fr
}

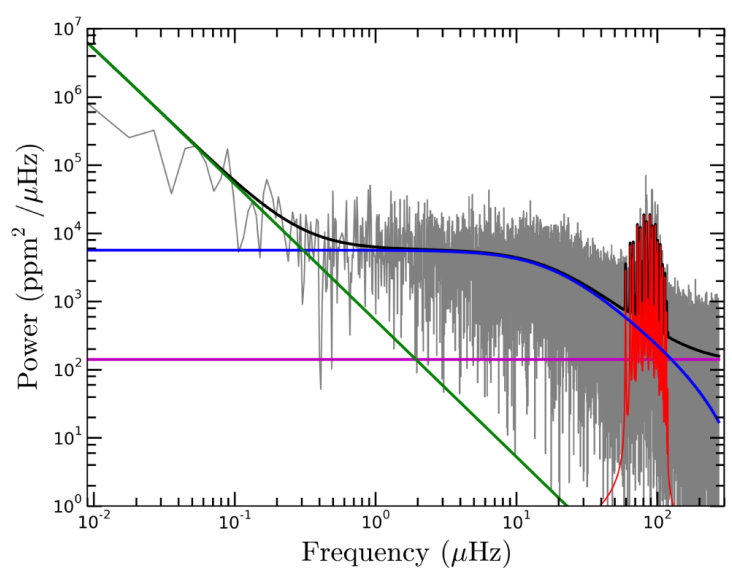

Figure 1: Result of the fit of the power density spectrum (PSD) associated with a Kepler red giant target (KIC 5527304).

\section{Website and search interface}

The SSI database and associated website is located at http: //ssi.lesia.obspm.fr. It provides access to the data and a brief description of the database and its content. An User Guide is also available for download.

Data retrieval is possible through the 'Search interface'. It allows the users to perform queries using star ID, star properties (e.g. $T_{\text {eff }}, \log$ g, spectral type, ...), and finally stellar indices (seismic indices and granulation parameters). A list of star ID can be provided by downloading a file containing these ID. Access to the data is also possible through a VO-compatible web service as well as through the Seismic Plus portal (http://voparis-spaceinn. obspm.fr/seismic-plus/). 

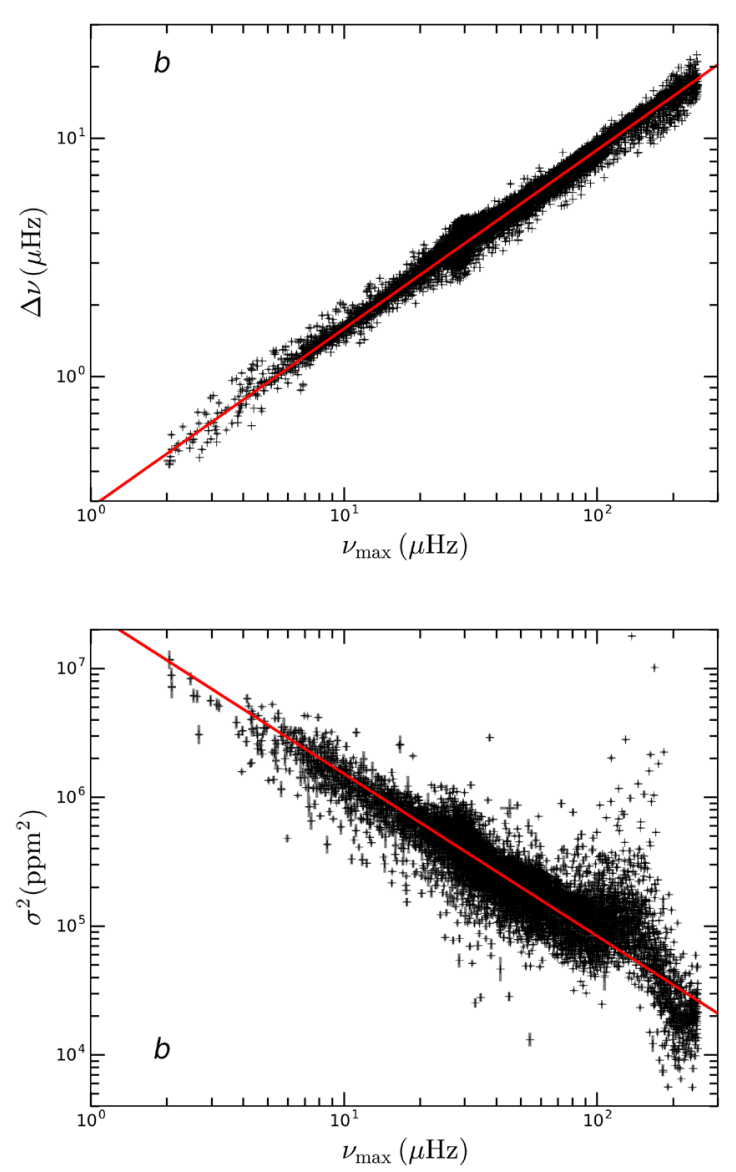

Figure 2: Top: $\Delta v$ as a function of $v_{\max }$ for about 13,000 Kepler red giants. Bottom: $\sigma^{2}$ as a function of $v_{\max }$.

\section{Query results and some illustrations}

The results of the request are displayed in the browser through a paginated data table (see the illustration of Fig. 3). They can be filtered and sorted by any parameters. All parameters are by default not displayed but by clicking on "Show/hide columns", it is possible to choose the parameters to be displayed. The results are displayed for a limited number of stars. The complete set can, however, be downloaded on demand in a CSV file.

The SSI pipeline was applied to about 114,000 stars observed by CoRoT in the exo- planet field and 210,000 stars observed during up to 4 years by Kepler. The corresponding light-curve were selected on the basis of their total duration (longer than 50 days) only, and accordingly without any prior concerning the evolutionary status of the targets. Stellar indices (seismic and granulation parameters) were then extracted for a total number of 18,000 red giants. Among the Kepler stars for which seismic indices were extracted, about 5,000 of them were not identified in the Kepler Input Catalog (KIC) as red giant. Some illustrative results are presented in Fig. 2.

The seismic indices $\Delta v$ and $v_{\max }$ obey characteristic scaling relations that depend directly on the radius, mass, and effective temperature of the star (for a review see e.g. [5]). From the knowledge of the effective temperature and these two seismic indices, it is then possible to estimate the mass and radius of the star. Note that a tool to derive stellar masses and radii from the seismic indices and effective temperatures is implemented in the Seismic Plus portal.

Concerning the granulation parameters, they also obey characteristic scaling relations that can provide informations about the stars in complement to the seismic indices [see e.g. 3, 4, 6, 7].

\section{Acknowledgements}

The Stellar Seismic Indices Data Base has been developed in the framework of the SPACEInn project (Exploitation of Space Data for Innovative Helio- and Asteroseismology), initiated by the European Helio- and Asteroseismology Network (HELAS) and financed by the European Union under the Seventh Framework Programme (FP7 project n. 312844). We acknowledge the Paris Data Centre for their financial and technical support. We thank S. Cnudde for the conception of the SSI logo and F. Henry for technical supports.

The CoRoT space mission has been developed and operated by CNES, with contributions from Austria, Belgium, Brazil, ESA (RSSD and Science Program), Germany, and Spain. Funding for the Kepler Discovery mission were provided by NASA's Science Mission Directorate.

\section{References}

[1] R.A. de Assis Peralta, R. Samadi, E. Michel, MNRAS, (submitted)

[2] B. Mosser, K. Belkacem, M.J. Goupil, E. Michel, Y. Elsworth, C. Barban, T. Kallinger, S. Hekker, J. De Ridder, R. Samadi et al., A\&A, 525, L9 (2011), 1011.1928

[3] S. Mathur, S. Hekker, R. Trampedach, J. Ballot, T. Kallinger, D. Buzasi, R.A. García, D. Huber, A. Jiménez, B. Mosser et al., ApJ, 741, 119 (2011), 1109. 1194

[4] T. Kallinger, J. De Ridder, S. Hekker, S. Mathur, B. Mosser, M. Gruberbauer, R.A. García, C. Karoff, J. Ballot, A\&A, 570, A41 (2014), 1408.0817

[5] K. Belkacem, Determination of the stars fundamental parameters using seismic scaling relations, in SF2A2012: Proceedings of the Annual meeting of the French Society of Astronomy and Astrophysics, edited by S. Boissier, P. de Laverny, N. Nardetto, R. Samadi, D. Valls-Gabaud, H. Wozniak (2012), pp. 173-188, arXiv:1210.3505, 1210.3505

[6] R. Samadi, K. Belkacem, H.G. Ludwig, A\&A, 559, A39 (2013), 1309. 1620

[7] R. Samadi, K. Belkacem, H.G. Ludwig, E. Caffau, T.L. Campante, G.R. Davies, T. Kallinger, M.N. Lund, B. Mosser, A. Baglin et al., A\&A, 559, A40 (2013), 1309. 1488 


\begin{tabular}{|c|c|c|c|c|c|c|c|c|c|c|c|c|c|c|}
\hline \multicolumn{2}{|c|}{ (4) Download as csv file } & \multicolumn{8}{|c|}{ Show $10 \vee$ entries } & \multicolumn{5}{|c|}{ Show / hide columns } \\
\hline \multirow{2}{*}{ StarID * } & \multirow{2}{*}{ Origin } & \multirow{2}{*}{$\begin{array}{l}\text { alpha } \\
\text { [deg] }\end{array}$} & \multirow{2}{*}{$\begin{array}{l}\text { delta } \\
\text { [deg] }\end{array}$} & \multirow{2}{*}{$\begin{array}{l}\text { teff } \\
\text { [K] }\end{array}$} & \multirow{2}{*}{$\underset{[\mathrm{cm} / \mathrm{s} 2]}{\text { grav }}$} & \multirow{2}{*}{ mag_v } & \multirow{2}{*}{ mag_r $\mathbf{x}$} & \multirow{2}{*}{ specType } & \multirow{2}{*}{ lumClass : } & \multicolumn{2}{|c|}{ numax_up $[\mu \mathrm{Hz}]_{\text {e }}$} & \multicolumn{3}{|c|}{ Deltanu_up $[\mu \mathrm{Hz}]$} \\
\hline & & & & & & & & & & value & prec & value & prec & $\Leftrightarrow 1$ \\
\hline 100402467 & corot & 290.570746 & 1.644686 & 4329 & & -99 & 14.12 & $\mathrm{~K}$ & II & 18.3 & 1 & 2.4912 & 0.012 & 8 \\
\hline 100412751 & corot & 290.586053 & 1.634669 & 4343 & & -99 & 13.43 & $\mathrm{~K}$ & III & 15.4118 & 0.586 & 1.9552 & 0.004 & 8 \\
\hline 100422482 & corot & 290.600554 & 1.717 & 4549 & & -99 & 12.13 & $\mathrm{~K}$ & III & 64.3284 & 0.963 & 6.0016 & 0.004 & 1 \\
\hline 100434740 & corot & 290.618651 & 1.62033 & 5031 & & -99 & 12.87 & G & $\mathrm{V}$ & 47.502 & 0.39 & 4.1872 & 0.008 & 8 \\
\hline 100440069 & corot & 290.626483 & 1.708309 & 4487 & & -99 & 12.87 & $\mathrm{~K}$ & II & 51.6 & 1 & 5.605 & 0.005 & 8 \\
\hline 100440565 & corot & 290.627243 & 1.647735 & 4507 & & -99 & 12.15 & K & II & 27.922 & 0.46 & 3.5088 & 0.004 & 1 \\
\hline 100441421 & corot & 290.628492 & 1.636505 & 4753 & & -99 & 12.89 & K & III & 32.94 & 0.3 & 4.4695 & 0.005 & 1 \\
\hline 100442460 & corot & 290.629967 & 1.714166 & 4549 & & -99 & 13.79 & $\mathrm{~K}$ & II & 28.4526 & 0.958 & 3.5328 & 0.006 & 1 \\
\hline 100448189 & corot & 290.638332 & 1.692805 & 4499 & & -99 & 13.53 & $\mathrm{~K}$ & II & 16.3917 & 0.467 & 2.3356 & 0.004 & 1 \\
\hline 100480626 & corot & 290.685877 & 1.647927 & 5673 & & -99 & 15.26 & $\mathrm{~F}$ & IV & 19.1542 & 0.689 & 2.1554 & 0.002 & 2 \\
\hline
\end{tabular}

Figure 3: Snapshot showing the query results 